\title{
Clinicoepidemiologic profile of patients with poisoning presenting to a tertiary care hospital; a one year preliminary descriptive study
}

\author{
Amir Mohammad Kazemifar ${ }^{1}$, Seyed Mostafa Mirakbari ${ }^{* \mathbb{C}^{\mathbb{D}}}$, Zohreh Yazdi², Bita Bitazar ${ }^{3}$, Pouria Soleimani ${ }^{4}$ \\ 'Department of Forensic Medicine and Toxicology, Bu Ali Hospital, Qazvin University of Medical Sciences, Qazvin, Iran \\ ${ }^{2}$ Department of Occupational Medicine, Bu Ali Hospital, Qazvin University of Medical Sciences, Qazvin, Iran \\ ${ }^{3}$ School of Medicine, Qazvin University of Medical Sciences, Qazvin, Iran \\ ${ }^{4}$ Student Research Committee, Qazvin University of Medical Sciences, Qazvin, Iran
}

\section{Correspondence to:}

Seyed Mostafa Mirakbari,

Email: drmirakbari@yahoo.com,

m.mirakbari@qums.ac.ir

Received: 21 July 2020 Accepted: 20 October 2020 ePublished: 13 Nov. 2020

Keywords: Drug overdose, Poisoning, Epidemiology, Mortality

\begin{abstract}
Introduction: Poisoning is regarded as a matter of concern for health providers as the burden of health resources loss can be high. Since nature and diversity of poisoning are different among various regions around the country, this study was conducted to demonstrate type and reasons for poisonings in Qazvin province, Iran.

Objectives: The aim of this study was to demonstrate the reason of toxic events as well as presenting symptoms and outcome of poisonings.

Patients and Methods: This prospective, cross-sectional and hospital-based study was conducted during a one-year period. The patients recruited to the study were consecutive intoxicated cases presenting to the hospital with alleged poisoning. Relevant epidemiological and clinical data from patients were collected and then analyzed using SPSS software.

Results: A total of 23161 patients were admitted to the hospital, out of which 766 (3.3\%) intoxicated patients were enrolled in the study. Mean age was 29.8 years old. Females outnumbered males but difference was not significant. The majority of the poisonings were deliberate (95.2\%). Main clinical features of poisoning were decreased consciousness (47.4\%), followed by lethargy (38.1\%) and nausea/vomiting (31.9\%). The benzodiazepines were the predominant agent ingested by patients, followed by opioids. Mortality rate was found to be $1 \%$.

Conclusion: Although the majority of poisoning cases are deliberate, however according to the type of pharmaceuticals involved in the poisonings and low-mortality rate, it is believed that the occurrence of the toxic events in many cases arises from impulsive behaviors originated from minor familial conflicts and incompetent life skills. Improving mental health and efficient coping skills can contribute to the reduction of intentional poisonings which in turn reduces the burden of hospital costs.
\end{abstract}

\section{Introduction}

Citation: Kazemifar AM, Mirakbari SM, Yazdi Z, Bitazar B, Soleimani P. Clinicoepidemiologic profile of patients with poisoning presenting to a tertiary care hospital; a one year preliminary descriptive study. J Prev Epidemiol. 2020;5(1):e15. doi: $10.34172 /$ jpe. 2020.15
Human poisoning is an emerging health problem worldwide that poses significant burden on emergency departments and quality of life (1-4). It is, particularly in terms of intentional attempt, frequently attributed to the problems associated with industrialized world in which the alarming increase in drug abuse and the phenomenal growth in the prescriptions and subsequent overdoses with mood-altering drugs are closely intertwined as a means to response to stressful events with little desire to die (5-7). A new approach has, however, been begun to specifically focus on the importance of this phenomenon in developing countries where urbanism and urban crowding

\begin{abstract}
Key point
Clinicoepidemiologic study of 766 intoxicated cases presented to the hospital revealed that the majority of toxic events were deliberate $(95.2 \%)$, however according to higher prevalence of low potent pharmaceuticals and low mortality rate $(1 \%)$, it is concluded that the events in most cases originate from minor social incompetencies or family conflicts leading to an impulsive intake of drugs. Thus, many intoxications are preventable by adequate psychological supports and life skill educations.
\end{abstract}

along with the lack of education systems in interpersonal communication skills and effective problem solving dominate parents, children and peers (6-8).

In 1991, Iran had the 91st rank of selfpoisoning in the world, which changed to

Copyright $(2020$ The Author(s); Published by Society of Diabetic Nephropathy Prevention. This is an open-access article distributed under the terms of the Creative Commons Attribution License (http://creativecommons.org/licenses/by/4.0), which permits unrestricted use, distribution, and reproduction in any medium, provided the original work is properly cited. 
58th in $2003(8,9)$. According to annual report of recorded phone calls to Iran's Drug and Poison Information Centers (2014-2015), 250368 phone calls were registered across the country (10). Toxicologic profiles including patterns of poisoning and the type of implicated poisons and morbidity and mortality rates varied by regions $(8,10)$. The persistent occurrence of deliberate self-poisoning poses an enormous challenge to health systems and would put limited resources at risk (7). As such of these evidences, the need for epidemiological studies should be regarded as highly important $(11,12)$.

Investigating the patterns of poisonings in a specified region contributes to early diagnosis and management of cases along with tailor-based preparedness of healthcare providers (13). This study aimed to depict the clinicepidemiologic profile of the cases admitted with alleged poisoning as well as to demonstrate the outcome of these cases.

\section{Objectives}

The aim of this study was to demonstrate the reason of toxic events as well as presenting symptoms and outcome of poisonings.

\section{Methods and Settings}

This study was conducted by investigating the sequential admitted cases with alleged poisoning and drug overdose presenting to the emergency department (ED) of Bu Ali hospital for one year starting March 21, 2011 to March 19, 2012. Data were collected through pre-designed forms and then analyzed using descriptive statistical methods.

$\mathrm{Bu}$ Ali hospital is an inner-city, 220-bed universityaffiliated tertiary care facility in Qazvin city of Qazvin province located in north-west of Iran. The province holds a population of 1300000 on the basis of 2016 census and is the hub of the first industrial zone of the country named Alborz city encompassing 2200 small to medium size operating factories. The ED cares for the majority of the province's acute adult poisonings and has been regarded as a referral center for complicated cases. The age groups of lower than 10 years old are referred to children hospital, thus were not investigated in this study.

\section{Ethical issues}

This study was extracted from the medical thesis of Bita Bitazar at emergency department of $\mathrm{Bu}$ Ali hospital approved by deputy of research (\#1011). All investigations performed in this study were in accordance with the ethical standards of the institutional and national research committee and with the 1964 Helsinki Declaration and its later amendments or comparable ethical standards. Informed consent was obtained from all individual participants involved in the study. The Ethics Committee of the Qazvin University of Medical Sciences approved the study.

\section{Data analysis}

For qualitative variables, mean \pm standard deviation (SD) was calculated. Frequency and percentages were calculated for qualitative variables. Chi-square test was used to compare qualitative variables and $P$ value $<0.05$ considered significant. Data analyses were performed using the Statistical Package for the Social Sciences (SPSS) software for Windows (SPSS Inc., Chicago, IL, USA) version 16 .

\section{Results}

During the one-year study period, a total of 23161 patients had been hospitalized in the hospital. Out of these admitted cases, 766 (3.3\%) cases were found to be intoxicated by drugs and chemicals. The epidemiologic and clinical characteristics of the poisoned patients has appeared in Table 1.

Females were affected more than males (52.3\% versus $47.7 \%)$. There was no significant difference regarding affected males versus females $(P>0.05)$. The predominant group with regard to marital status was the married (52.2\%). 95.2\% of intoxications was attempted suicide (intentional). Age range varies from 10 to 90 years of old (mean 29.8). The number of admissions was significantly higher in the first half of year (592 cases) than those in the second half of year (174) (Figure 1). The most common

Table 1. Characteristics of admitted patients with alleged poisoning $(\mathrm{N}=766)$

\begin{tabular}{lcc}
\hline Parameter & No. & $\%$ \\
\hline Age (Mean) & 29.8 & - \\
Gender & & \\
$\quad$ Male & 356 & $(47.7)$ \\
$\quad$ Female & 401 & $(52.3)$ \\
Marital status & & \\
Married & 400 & $(52.2)$ \\
Single, divorced, separated, widowed & 366 & $(47.8)$ \\
\hline Time of intoxication to admission (Mean \pm SD) & $2.5 \pm 2.8$ & - \\
Presenting symptoms & & \\
Lethargy & 292 & $(38.1 \%)$ \\
Nausea/vomitting & 244 & $(31.9 \%)$ \\
Decreased LOC & 363 & $(47.4 \%)$ \\
Asymptomatic & 129 & $(16.8 \%)$ \\
Psychiatric co-morbidity & 181 & $(22.6 \%)$ \\
Organic disabilities or illnesses & 118 & $(15.4 \%)$ \\
Substance abuse & 309 & $(40.3 \%)$ \\
Mortality & 8 & $(1 \%)$ \\
\hline Place of residence & & \\
Urban & 589 & $(76.9 \%)$ \\
Rural & 177 & $(23.1 \%)$ \\
\hline LOS (day) & 368 & $(48 \%)$ \\
\hline Disposition & & \\
Medical discharge & & \\
Discharge against medical advice & & \\
\hline
\end{tabular}

LOC, level of consciousness; LOS, length of hospital stay. 


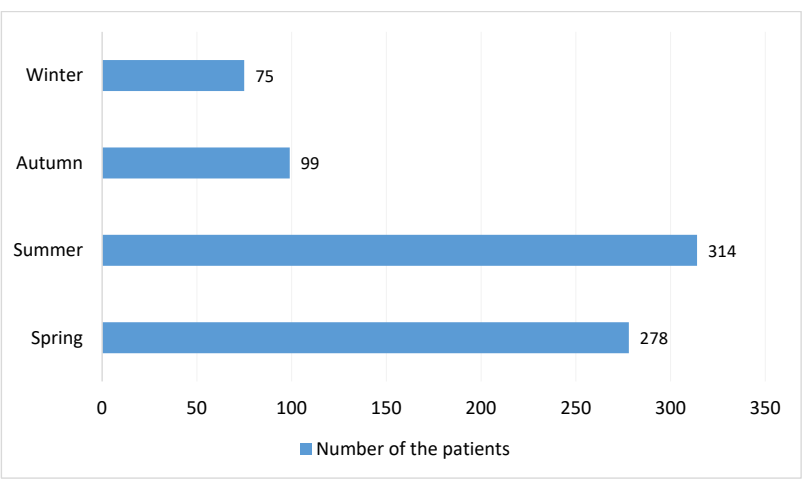

Figure 1. Seasonal distribution of poisonings $(\mathrm{N}=766)$.

clinical finding on admission was decrease in level of consciousness (47.4\%), followed by lethargy (38.1\%). Around $40.3 \%$ of the cases were suffering from substance abuse $(40.3 \%)$, followed by psychiatric co-morbidities (22.6\%) and organic illnesses (15.4\%). A detailed breakdown of exposures has appeared in Table 2. $48 \%$ of cases were discharged against medical advice. Mortality rate was discovered to be $1 \%$.

\section{Discussion}

Poisoning is one of the major burden of costs as well as time consuming load of nursing work in the country $(8,9)$. The nature and diversity of poisonings differs on the basis of geographic, cultural and socioeconomic background of the societies $(7,8)$. Thus, our preliminary study was warranted to be conducted in the province of Qazvin where it is recognized as an industrial-oriented province.

This study revealed that females are slightly more susceptible than males. Male-to-female ratio was 1:1.1. This result corresponds with the study findings conducted in north and north-east cities of the country (14). However, it is inconsistent with the data obtained in the study performed in Shahrekord (west city of Iran), in which the males have been affected slightly more (15).

Greenberg et al have noted seasonal trends in reported poisonings (16). In our study, most of exposures have occurred in the spring and summer (77.3\%) (Figure 1). In the study conducted by Mehrpour et al, $50.2 \%$ of poisonings have occurred in these seasons (17). The same pattern of hot season dominance of poisoning is seen in the study conducted by Ahmadi et al in Sari, north of Iran (14). However, it has been shown that the nature and type of poisoning differs in regard to hot versus cold seasons $(8,14)$.

The mean time of arrival at emergency care was 2.5 hours that means the public awareness along with transportation facilities works enough in this province. In the study by Ahmadi et al, the majority of the patients were transferred to the hospital in 2-6 hours after poisoning.

The most commonly presenting symptoms were decreased level of consciousness $(47.4 \%)$, followed by lethargy (38.1\%). It seems the poly-drug intoxications may
Table 2. Comparison of the type of poison/drug exposure $(\mathrm{N}=766)$

\begin{tabular}{lc}
\hline Type of poison/drug & No. $(\%)$ \\
\hline Psychotropics & $77(10.1)$ \\
\hline Analgesics & $89(11.6)$ \\
\hline Alcohol & $2(0.3)$ \\
\hline Benzodiazepines & $182(23.8)$ \\
\hline Barbiturates & $36(4.7)$ \\
\hline Household cleaning products & $6(0.8)$ \\
\hline Agrochemicals* & $26(3.4)$ \\
\hline Antihistamines & $3(0.4)$ \\
\hline Opioids & $149(19.5)$ \\
\hline Unknown & $196(25.6)$ \\
\hline *Pesticides and herbicides
\end{tabular}

contribute to these higher clinical manifestations. A study in Saudi Arabia delivered the similar result with symptoms of the central nervous system in $57.4 \%$ of cases (18).

A total of $390(51 \%)$ cases were discharged with recovery and $368(48 \%)$ patients denied the continuation of treatment and left the hospital against medical advice. Benzodiazepines were the most commonly used group of pharmaceuticals exposures in our patients and is consistent with other studies $(14,17)$. We think that benzodiazepine intoxications have happened in a situation of familial conflicts with subsequent impulsive intake that eventually has led to a minimal toxicity with a drug with easy availability, contributing to lower mortality rate and more discharge rate against medical advice. The mortality rate was found to be $1 \%$ ( 8 cases). The mortality rate in the study by Ahmadi et al (14) and the study conducted by Mehrpour et al (17) was $1.3 \%$ and $19.5 \%$, respectively. The mortality rate is dissimilar in different studies and may be subject to study population, the type of drug used by the patients, the introduction of new drugs and chemicals and location of the study (8).

This is the first study in this province that initially explores extent and context of issues related to referral intoxications. Furthermore, it uncovers a portfolio that can be measured as a key feature for establishing preventive strategies and ongoing future studies.

\section{Conclusion}

The pharmaceuticals, particularly benzodiazepines, remain as the most commonly encountered toxic exposures that depict a toxic profile with a readily available drug category. This pattern shows that the event of poisonings in most cases could easily be declined through adequate family educations and raising social awareness along with maintaining healthy life skills and implementing psychologic supports in vulnerable individuals. Opioid overdoses encompassed the most second group of intoxications that urges the endeavors tackling substance abuse and focusing on interventions in harm reduction programs. 


\section{Limitations of the study}

This is a simple descriptive study from one area of Iran with modest international importance. It presents information from a single hospital and has no intervention.

Acknowledgements

We would like to thank the administration and staff of Qazvin University of Medical Sciences for their assistance in performing the study.

Conflicts of interest

The authors declare that there are no conflicts of interest.

Authors' contribution

AMK and ZY designed the research study; BB collected data; ZY and SMM analyzed the data; SMM and PS wrote the paper.

Funding/Support

The study did not have any financial supporters.

\section{References}

1. Camidge DR, Wood RJ, Bateman DN. The epidemiology of self-poisoning in the UK. Br J Clin Pharmacol. 2003;56(6):6139 .

2. Assadi R, Van Spijker BAJ, Smit F, Afshari R. Preference-based health valuation for acute poisoning: the challenge of very acute disease and rapid reversal of the health state. Iran Red Crescent Med J. 2017;19:e24386

3. Van Hoving D, Hunter L, Gerber R, Lategan H, Marks C. The burden of intentional self poisioning on a district-hospital public hospital in Cape Town, South Africa. Afr J Emerg Med. 2018;8:79-83.

4. Mew EJ, Padmanathan P, Konradsen F, Eddleston M, Chang SS, Phillips MR, et al. The global burden of fatal selfpoisoning with pesticides 2006-15: systematic review. J Affect Disord. 2017;219:93-104.

5. Eddleston M, Gunnell D, Karunaratne A, de Silva D, Sheriff $\mathrm{MH}$, Buckley NA, et al. Epidemiology of intentional self- poisoning in rural Sri Lanka. Br J Psychiatry. 2005;187:583-4. doi: 10.1192/bjp.187.6.583.

6. Chowdary AN, Banerjee S, Brahma A, Biswas MK. Pesticide poisoning in nonfatal, deliberate self-harm: A public health issue. Indian J Psychiatry 2007;49:117-20.

7. Eddleston M. Patterns and problems of deliberate selfpoisoning in the developing world. QJM. 2000;93(11):715-31.

8. Alinejad S, Zamani N, Abdollahi M, Mehrpour O. A Narrative Review of Acute Adult Poisoning in Iran. Iran J Med Sci. 2017;42(4):327-346.

9. Nikfar S, Khatibi M, Abdollahi-asl A, Abdollahi M. Cost and utilization study of antidotes. Int J Pharmacol 2011;7:46-49

10. Ghane T, Behmanesh Y, Khazaei F. Annual report of recorded phone calls to iran's drug and poison information centers (2014-2015). Asia Pac J of Med Toxicol. 2015;4(3):97-101.

11. Singh S. Research Priorities for Medical Toxicology in Low and Middle Income Countries. Asia Pac J Med Toxicol. 2013;2(2):78.

12. Afshari R. What is the "Best Research" for Low Income Countries? Asia Pac J Med Toxicol. 2013;2(1):1.

13. Forging a Poison Prevention and Control System: Data and Surveillance. ashington (DC): National Academies Press (US); 2004.

14. Ahmadi A, Pakravan N, Ghazizadeh Z. Pattern of acute food, drug, and chemical poisoning in Sari City, Northern Iran. Hum Exp Toxicol. 2010;29:731-8.

15. Akhlaghi M, Arbabi Z, Khadivi R. Pattern of acute poisoning in Shahrekord (Western Iran). Asian J Epidemiol. 2009;2:9-12.

16. Greenberg RS, Osterhout SK. Seasonal trends in reported poisonings. Am J Public Health. 1982;72:394-6.

17. Mehrpour O, Akbari A,Jahani F, et al. Epidemiological and clinical profiles of acute poisoning in patients admitted to the intensive care unit in eastern Iran (2010 to 2017). BMC Emerg Med. 2018;18:30. doi: 10.1186/s12873-018-0181-6.

18. Bakhaidar M, Jan S, Farahat F, et al. Pattern of drug overdose and chemical poisoning among patients attending an emergency department, western Saudi Arabia. J Community Health. 2015:40:57-61. 